\title{
Daten, das Öl des 21. Jahrhunderts
}

\section{Christoph Bosshard}

Dr. med., Vizepräsident der FMH, Mitglied des Zentralvorstandes der FMH, Departementsverantwortlicher Daten, Demographie und Qualität / Schweizerische Akademie für Qualität in der Medizin SAQM

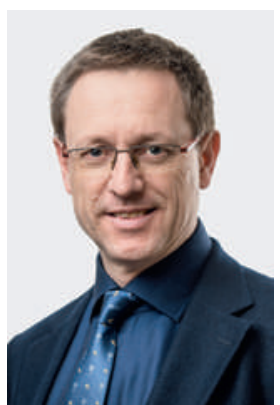

Auch wenn uns der Kalender Schritt um Schritt in Richtung Frühling führt, so herrschen draussen nach wie vor winterliche Temperaturen. Wer über die notwendigen Energiereserven verfügt, muss nicht schlottern. Gut auch, wer über genügend eigene Daten verfügt, um sich für die diesbezüglichen Herausforderungen zu wappnen.

Die ärzteeigenen Daten ermöglichen es der FMH, auch dieses Jahr wieder statistische Grundlagen von hohem Wert in die gesundheitspolitischen Diskussionen einzubringen - nicht um diese anzuheizen, sondern um diese mit konstruktiver Energie zu versorgen. Alle reden von Steuerung in unserem Gesundheitswesen. Die FMH zeigt währenddessen auf, wo und wie ihre rund 40000 Mitglieder rudern und damit dazu beitragen, die Gesundheitsversorgung in der Schweiz auf Kurs zu halten. Diese Daten helfen mit, den Verantwortungsträgerinnen und -trägern für ihre nicht leichte Arbeit Grundlagen zu geben.

Die klaren Tendenzen bezüglich Entwicklung der Teilzeitarbeit und der Altersstruktur der Ärzteschaft lassen unweigerlich aufhorchen. Die in den verdienten Ruhestand übertretenden Kolleginnen und Kollegen können wir nur noch ersetzen, wenn wir jedes Jahr zunehmend mehr Ärztinnen und Ärzte aus unseren Nachbarländern in die Schweiz holen. Abgesehen von den moralisch schwierigen Folgen dieses Brain-Drains, wenn den Regionen am anderen Ende der Welt die Fachkräfte fehlen, stellt sich die Frage, ob in absehbarer Zukunft diese Fachleute über-

Mit ihren statistischen Grundlagen versorgt die FMH die gesundheitspolitischen Diskussionen mit konstruktiver Energie.

haupt noch verfügbar sein werden. Einerseits herrscht ein globaler Ärztemangel, andererseits haben unsere Nachbarländer begonnen, ebenfalls in die Attraktivität ihrer Rahmenbedingungen zu investieren. Daher sei die Prognose erlaubt: Ohne Massnahmen werden uns die Fachkräfte ausgehen, bevor uns das Geld ausgeht! Es braucht also innovative Ideen. Die Erhöhung der Anzahl Studienplätze ist unabdingbar, wirkt jedoch nach einem sechsjährigen Medizinstudium und einer daran anschliessenden ebenso langen Facharzt-Wei- terbildung frühestens zwölf Jahre nach Beginn der Umsetzung dieser Massnahme. Was könnten wir bis dahin tun? Mit Blick auf die zunehmende TeilzeitTätigkeit und Feminisierung sind Angebote zu schaffen, welche den Bedürfnissen der kommenden Generationen gerecht werden. Nur so werden wir es schaffen, unsere nachfolgenden Kolleginnen und Kollegen zu einem möglichst hohen Beschäftigungsgrad-Anteil zu motivieren. Zusätzlich könnte aktuell brachliegendes Potential mobilisiert werden, indem nicht mehr ärztlich Berufstätige zum Wiedereinstieg ermuntert werden. Eine solche Entwicklung ist nicht nur für das Gesundheitswesen sinnvoll, sondern für die gesamte Volkswirtschaft. Es kann ja wohl kaum sein, dass wir als Gesellschaft in die Bildung unserer Bürgerinnen und Bürger investieren und ihre Schaffenskraft dann nicht nutzen wollen. Wenn in einer Partnerschaft beide berufstätig sind, so entstehen neue Rollen und Herausforderungen - geschlechts- und berufsunabhängig.

\section{Ohne innovative Massnahmen werden uns die ärztlichen Fachkräfte ausgehen, bevor uns das Geld ausgeht!}

Dass es auch in der Medizin schwierig sein wird, das Ist zum künftigen Soll zu erheben, macht nicht nur die demographische Entwicklung unserer Bevölkerung deutlich. Mit den Jahren kommen diverse gesundheitliche und allenfalls auch soziale Herausforderungen zusammen, welche plötzlich gemeinsam Krankheitswert erreichen können. Nicht nur so ist der Wandel zu sehen, sondern auch ganz fachspezifisch gesehen eröffnen sich heute zum Beispiel im Bereich des Herzklappen-Ersatzes katheterbasierte Möglichkeiten, in welchem es früher die Chirurgie brauchte. Wer weiss, welche Innovationen uns die Zukunft noch bereithält? Welche Ressourcen wird unsere Gesellschaft in ihr Gesundheitswesen investieren wollen und können? Vor diesem Hintergrund relativieren sich die rein datenbasierten Steuerungsmöglichkeiten und fordern uns Ärztinnen und Ärzte auf, uns aktiv an diesen Diskussionen zu beteiligen. Auch hier braucht es wie beim Rudern den Beitrag von allen Crewmitgliedern. Ich danke Ihnen dafür! 